\title{
Genetics of divergence in male wing pigmentation and courtship behavior between Drosophila elegans and $D$. gunungcola
}

\author{
S-D Yeh, S-R Liou and JR True \\ Department of Ecology and Evolution, State University of New York at Stony Brook, 650 Life Sciences Bldg., Stony Brook, \\ NY 11794-5245, USA
}

\begin{abstract}
Many sex-specific traits involved in mating consist of functionally coordinated morphologies and behaviors. How the components of these complex traits evolve and become coordinated during evolution is unknown. In order to understand how such trait complexes evolve and diversify, we must decipher the genetic underpinnings of their components. In this study, we begin to elucidate the genetic architecture underlying differences in functionally related male pigmentation and behavior between two Asian Drosophila melanogaster group species, $D$. elegans and $D$. gunungcola. $D$. elegans possesses a male-specific wing melanin spot and a stereotypical wing display element in male courtship, whereas $D$. gunungcola lacks both of these traits. Using reciprocal F1 male hybrids, we demonstrate that
\end{abstract}

the X-chromosome contains a major locus or loci required for wing spot formation and that autosomal loci largely determine the male courtship display. Using phenotypic and genetic analysis of backcross progeny, we further demonstrate that both the wing spot and courtship differences between the two species are polygenic and both depend at least in small part on genetic factors on both the $X$ and the autosomes. Finally, we find that male wing spot size and courtship wing display are highly correlated in backcross progeny, suggesting that linkage or pleiotropy may have been involved in their coordinated evolution.

Heredity (2006) 96, 383-395. doi:10.1038/sj.hdy.6800814; published online 29 March 2006

Keywords: sexual selection; melanin patterns; premating isolation; courtship behavior; large X-effect; correlated characters

\section{Introduction}

In animals, many obvious species differences consist of complex combinations of male-specific ornamentation and behavioral characters presumed to have evolved via sexual selection (Andersson, 1994). These traits are often proposed to be involved in sexual isolation during speciation (Lande, 1981; West-Eberhard, 1983; Kaneshiro and Boake, 1987; Higashi et al, 1999; Kaneshiro, 2000; Panhuis et al, 2001). Selection promoting combinations of visible traits and behavior presumably requires both components to be present. For example, both transverse wing bands and wing flicking behavior are required for Zonosemata vittigera flies to escape from the predation of salticid spiders (Greene et al, 1987; Whitman et al, 1988). Therefore, the traits are expected to evolve in concert (eg Prum, 1990, but see Wiens, 2000 for a counterexample).

Little is known about the genetic mechanisms by which natural and sexual selection construct such functionally related trait combinations. Are independent sets of genes involved in each component trait? Are the genes involved in separate components, for example, a novel behavior and a novel morphology, linked in the

Correspondence: JR True, Department of Ecology and Evolution, State University of New York at Stony Brook, 650 Life Sciences Bldg., Stony Brook, NY 11794-5245, USA.

E-mail: jrtrue@life.bio.sunysb.edu

Received 28 September 2005; accepted 20 February 2006; published online 29 March 2006 genome and is this linkage important for the long-term evolution of the adaptation? Do some genes play roles in multiple traits? Geneticists have recently begun to address these questions. For example, Hawthorne and Via (2001) used quantitative trait locus (QTL) mapping to examine the architecture of genetic correlations between a behavioral trait, host choice, and a physiological trait, performance on the host, in the aphid Acyrthosiphon pisum. They found that the QTL effects underlying these two distinct traits were not distributed randomly in the genome. QTLs tended to be clustered, with each cluster containing QTL effects consistent with adaptation to one of the two hosts. More empirical studies are needed in order to determine whether such functionally related QTL clusters, which represent linked or pleiotropic loci, are a general feature of adaptive evolution.

In this study, we begin to decipher the genetic architecture of coordinately evolving male pigmentation and behavioral traits in the sibling species pair Drosophila elegans and D. gunungcola, which are members of the elegans subgroup of the melanogaster species group (Hirai and Kimura, 1997; Kimura and Hirai, 2001). D. elegans is distributed throughout Southeast Asia, including Malaysia, Philippines, Hong Kong, Taiwan, and Okinawa. D. gunungcola is found in Indonesia and its range overlaps at least partially with $D$. elegans (Hirai and Kimura, 1997; Sultana et al, 1999; Ishii et al, 2002; Suwito et al, 2002). D. elegans and D. gunungcola court each other in the laboratory and interspecific courtships have been 
reported in the field (Ishii et al, 2002). However, interspecific copulation or production of hybrids has not been reported.

The Oriental melanogaster group species vary in a number of male secondary sexual traits (Kopp and True, 2002a), the most obvious of which is the presence of a male-specific wing melanin spot, which takes on various shapes and intensities in several Oriental species (Figure $1 \mathrm{a}-\mathrm{c})$. The Oriental species also differ strikingly in male courtship. All of these species exhibit standard elements of Drosophila male courtship, including orientation and following, wing extension, and licking (Greenspan and Ferveur, 2000). However, species with male-specific wing spots, including $D$. elegans, exhibit a qualitative difference in courtship in comparison to nonspot-bearing species. In the spot-bearing species, the male exhibits a conspicuous wing display behavior in which he circles to the front of the female and faces her, then repeatedly waves his wings and shakes his body. D. elegans males may also extend their wings while competing with other males for territories on flowers (Kimura and Hirai, 2001). Species lacking wing spots, including D. gunungcola, may face the females while performing courtship song, but they do not perform the conspicuous wing display of the spot-bearing species in either courtship or male-male interactions. The strong association of wing spots with wing displays in this species group (Figure 1a) suggests that the two traits are maintained for a joint function, and that sexual selection, involving either female choice or male-male interactions, has been important in maintaining these two traits. The sibling species pair D. elegans and D. gunungcola provide an excellent opportunity to study the genetics of pigmentation and courtship evolution because they are the only known interfertile pair of species in this group that differ qualitatively in the presence of male wing spots and wing displays. Here, we present an initial analysis of the genetic basis of male wing spot and courtship divergence between $D$. elegans and D. gunungcola, using reciprocal F1 crosses and backcrosses with molecular marker genotyping.

\section{Materials and methods}

\section{Drosophila strains and cultures}

D. elegans HK (collected in Hong Kong), a brown morph strain and D. gunungcola SK (collected in Sukarami, Indonesia), were kindly provided by Dr Masahito $\mathrm{T}$ Kimura. Cultures were kept on standard fly food (corn meal, yeast extract, and agar) with folded paper inserted into the media for pupation.

\section{Drosophila crosses}

For all crosses, virgin females and males were collected within $6 \mathrm{~h}$ after eclosion and housed separately in food vials for 3-5 days. For hybrid crosses, five to 10 virgin females from one species and five to 10 virgin males from the other species were put in a vial with food and transferred into a new vial every week for several weeks until all the females were dead. After eclosion, hybrid progeny were sorted and stored in separate vials by sex for $3-5$ days. The F1 hybrid females were then backcrossed to either parental species males while the F1 hybrid males and backcross progeny were either preserved in $70 \%$ ethanol or frozen after their courtship behavior was observed.
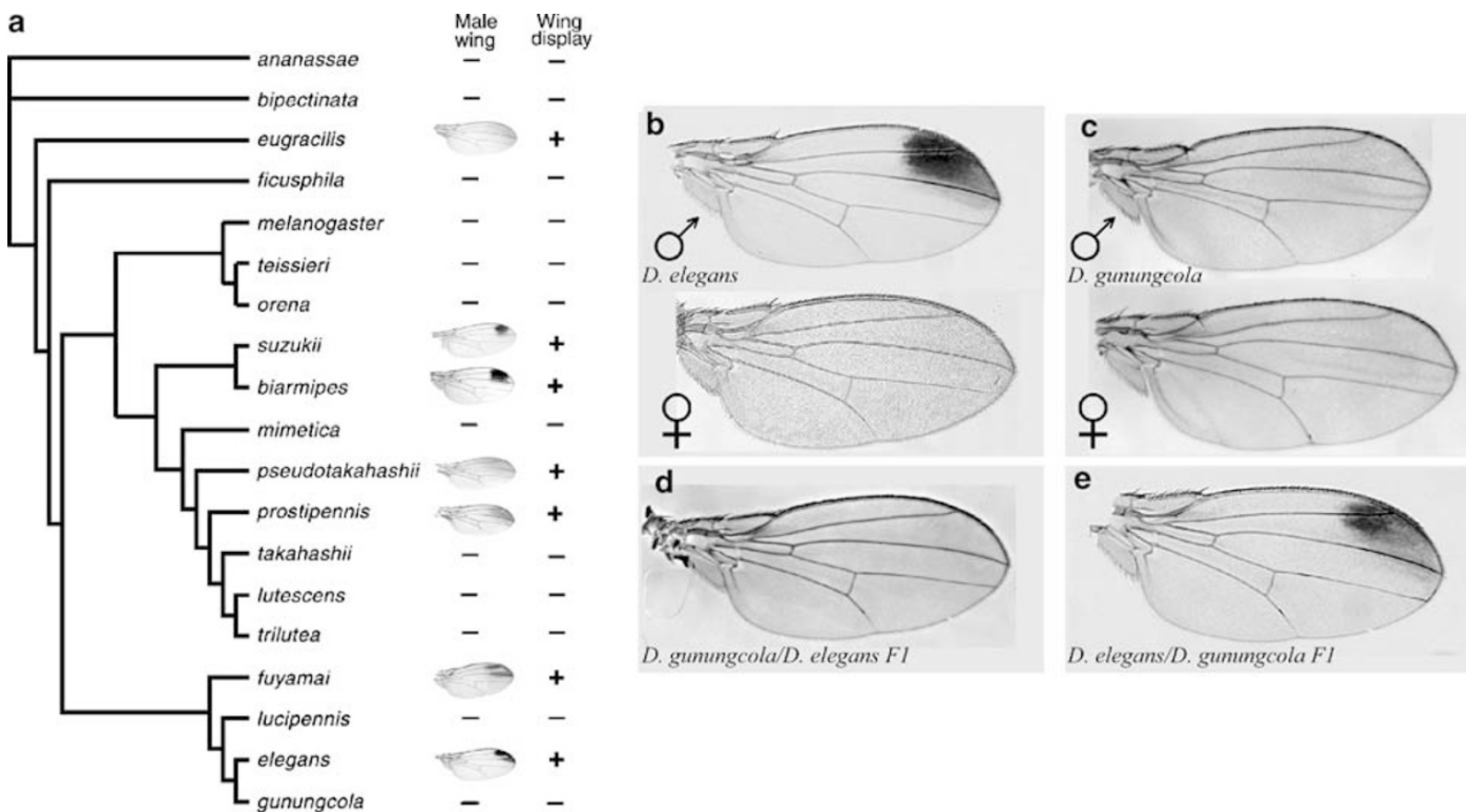

Figure 1 (a) Phylogeny of Oriental D. melanogaster group species (after Kopp and True, 2002a, b) showing typical male wing of species bearing male wing spots. In all species except D. eugracilis, female wings have no pigmentation. In D. eugracilis, both male and female wings have slight anterior-distal melanization (male shown). Plus signs indicate species possessing wing displays. Dashes indicate species lacking wing spots and/or wing displays. (b) D. elegans male and female wings. (c) D. gunungcola male and female wings. (d) Typical wing of a D. gunungcola/D. elegans F1 male hybrid carrying a D. gunungcola X-chromosome and a D. elegans Y-chromosome. (e) Typical wing of a D. elegans/ D. gunungcola F1 male hybrid carrying a D. elegans X-chromosome and a D. gunungcola Y-chromosome. 


\section{Genotyping methods}

Genomic DNA of male backcross progeny was isolated by grinding individual flies in $0.2 \mathrm{mg} / \mathrm{ml}$ proteinase $\mathrm{K}$ in homogenization buffer $(50 \mathrm{mM}$ Tris- $\mathrm{HCl}, \mathrm{pH} 7.5,20 \mathrm{mM}$ EDTA, $0.5 \%$ SDS) followed by incubation at $55^{\circ} \mathrm{C}$ for $2 \mathrm{~h}$. $\mathrm{NaCl}$ was then added to $400 \mathrm{mM}$ final concentration and preps were centrifuged at 14000 r.p.m. for $10 \mathrm{~min}$. DNA in supernatant was then ethanol-precipitated. Pellets were air-dried and resuspended in $50 \mu 110 \mathrm{mM}$ Tris- $\mathrm{HCl}$, $\mathrm{pH}$ 8.5. Preps were diluted 100-fold and 1-10 $\mu \mathrm{l}$ of dilution was used as template in each PCR reaction.

The primers used to amplify target loci were designed in conserved regions determined by comparison of the $D$. melanogaster and D. pseudoobscura genomes. More specific primers for genotype diagnosis in male backcross progeny were designed based on DNA sequence of D. elegans and D. gunungcola (see Table 1). Initially, primers for five genes, yellow (y), aristaless (al), Ecdysone receptor $(E c R)$, aracaun (ara), and ebony (e) were developed, providing markers corresponding to each major chromosome arm of $D$. melanogaster (the effect of the tiny fourth chromosome, which constitutes about $2 \%$ of the genome, was not studied). These five markers were all unlinked in D. elegans/D. gunungcola (data not shown), which is consistent with their positions on different chromosome arms, but this has not yet been confirmed by physical mapping. Two further markers, Moesin-like (Moe), which is close to pigmentation candidate gene tan on the D. melanogaster X-chromosome and transcription factor II A-L (TfIIA-L) on the right arm of the $D$. melanogaster third chromosome, close to $e$, were also developed. D. elegans HK- and D. gunungcola SK-specific alleles were defined either by single-nucleotide polymorphisms (SNPs), genotyped using restriction enzyme digestion followed by agarose gel electrophoresis, or insertion/deletion differences, genotyped by gel electrophoresis alone. Methods and primers used for each marker locus are summarized in Table 1.

The lack of obvious inversion loops in the salivary gland chromosomes of female D. elegans HK/D. gunungcola SK F1 hybrid larvae (JR True, unpublished) indicates that D. elegans and D. gunungcola are homokaryotypic on a gross scale. Small inversion differences between $D$. elegans and D. gunungcola, constituting less than half of a chromosome arm, cannot be ruled out at this time, but such differences, if present, would not affect the conclusions of this study.

Although the cytological map of genes in the D. elegans species subgroup is not available, the polytene chromo- somal structure of these two species is very similar to $D$. melanogaster, with five major chromosome arms (JR True, unpublished). In this study, we use genetic markers presumed to be homologous to $D$. melanogaster loci. Results are depicted using the approximate physical positions of the $D$. melanogaster homologs, although this has been established only for the X-linked markers, $y$ and Moe. $y$ is allelic to a spontaneous mutant in D. elegans that is null for Yellow protein expression in 60-75 h old pupae and bears light yellow body pigmentation similar to $D$. melanogaster (JR True and S-D Yeh, unpublished data). Chromosome arm assignments of the autosomal markers are tentative, but because they are all unlinked, their coverage of the D. elegans and D. gunungcola genomes is approximately the same as one marker per major chromosome arm.

\section{Statistical analyses}

Genotype-phenotype data were analyzed using JMP version 4.04 software (SAS Institute, Cary, NC, USA). For the analysis of individual courtship elements, individual male backcross progeny were classified as either exhibiting or not exhibiting each of four courtship elements. These data were coded nominally and tested for associations with genotype at each marker separately using a nominal logistic test in JMP. Trait correlations in backcross progeny were assessed using the nonparametric Kendall's coefficient of rank-correlation ( $\tau$; Sokal and Rohlf, 1995) in JMP.

\section{Behavioral and pigmentation assessment in male $\mathrm{F} 1$ and backcross progeny}

Male backcross progeny were obtained by backcrossing the F1 hybrid females to males of either parental species. Virgin females and males were collected using light $\mathrm{CO}_{2}$ anesthetization and stored separately (in groups of 5-20) in food vials $3-5$ days after eclosion in a $25^{\circ} \mathrm{C}$ incubator with a 12:12 h light:dark cycle before behavioral observation. Flies were then individually separated into food vials, again using light $\mathrm{CO}_{2}$ anesthetization at least $24 \mathrm{~h}$ but no more than $72 \mathrm{~h}$ before the courtship observations were performed. The courtship behavior of individual males was observed by placing the male in a food vial containing one D. elegans $\mathrm{HK} 3$ - to 5-day-old virgin female and one D. gunungcola SK 3- to 5-day-old virgin female in a vial without anesthesia. The courtship behavior of each male was observed until copulation occurred or $1 \mathrm{~h}$ elapsed. In order to make sure male

Table 1 Molecular marker primer sets, annealing temperatures, and alleles used in this study

\begin{tabular}{|c|c|c|c|c|}
\hline Locus & Forward primer sequence & Reverse primer sequence & $\begin{array}{l}\text { Anneal. } \\
\text { temp. } \\
\left({ }^{\circ} \mathrm{C}\right)\end{array}$ & $\begin{array}{l}\text { Allelic difference between } \\
\text { D. elegans } H K \text { and } \\
\text { D. gunungcola } S K\end{array}$ \\
\hline$y$ & 5'-CCCAGCCCATACССТTTCAAAAATG-3' & 5'-AATCCTCTTCTGTGGACCGTGGCGC-3' & 60 & $\begin{array}{l}\text { Indel: } 43 \mathrm{bp} \text { deletion in } \\
\text { D. elegans HK }\end{array}$ \\
\hline Moe & 5'-CCGKAAYACATTCAAGTATGG-3' & 5'-AGATCCTGTTTCAGGGCCTGAA-3' & 56 & $\begin{array}{l}\text { Indel: } 121 \mathrm{bp} \text { deletion } \\
\text { in D. elegans HK }\end{array}$ \\
\hline al & 5'-GAGAATTCAGGGGCTCCAAGCTG-3' & 5'-AACTGACCGGGCATGTAATGAC-3' & 66 & $\begin{array}{l}\text { Indel: } 15 \text { bp deletion in } \\
\text { D. gunungcola SK }\end{array}$ \\
\hline$E c R$ & 5'-AGAGGATCTCAGGCGTATAATG-3' & 5'-CMGCCATTCCGGCCATTTTGTA-3' & 56 & RFLP: MluI \\
\hline ara & 5'-GYGAGAAGATYATGCTGGCCAT-3' & 5'-ATGGCATCCTCCTCCTCTTTGG-3' & 55 & RFLP: ScrF1 \\
\hline$e$ & 5'-AAGTGCATGCAGGCGATGTTCTCG-3' & 5'-GGTGGCCAGTAACCAGACTTGATTCT-3' & 57 & RFLP: ScaI \\
\hline TfIIA-l & 5'-CGCATTCTTGTGCCATTTGTATG-3' & 5'-ATGGCTTTACCTTGGTGCTCTG-3' & 56 & RFLP: MslI \\
\hline
\end{tabular}


Table 2 (a) Courtship categories in D. elegans/D. gunungcola backcross hybrids and (b) courtship elements investigated in this study

\begin{tabular}{ll}
\hline Score & Description \\
\hline (a) Courtship categories in D. elegans/D. gunungcola backcross hybrids \\
Male stays beside or behind female at all times during courtship. No circling is seen, and no double wing extensions \\
over $45^{\circ}$ are performed, like a D. gunungcola male. \\
Male moves from the back of the female to the side or nearly (but not directly) in front of the female several times. Wing \\
waving, in which one or both wings are moved by flexing of wing hinges, occurs and wing may be raised above body \\
instead of spread outward. Body shaking occurs during wing movements. Note that wing waving is distinct from wing \\
display (see Table 2b).
\end{tabular}

behavior was thoroughly tested, each male was observed on the next day with one new virgin female of each species. Males differing in courtship score over the 2 days were generally rare and were assigned the more D. elegans-like of the two scores (higher score; see below). This is because we were interested primarily in the ability of individual males to perform particular courtship elements, rather than the frequency at which they performed them. D. elegans courtship is more elaborate than D. gunungcola (ie it consists of more elements). The ability of backcross males to perform these elements is expected to be determined by the presence of $D$. elegans-specific alleles and the lack of ability to perform these elements is expected to be determined by the presence of D. gunungcola-specific alleles. Males were preserved in $1.5 \mathrm{ml}$ microcentrifuge tubes individually and frozen at $-20^{\circ} \mathrm{C}$ before dissection and DNA isolation.

The courtship score of each backcross male was determined by observing the courtship elements that male exhibited using a scale of 1-4. The specific criteria for each courtship score are listed in Table 2a. The presence or absence of three D. elegans-specific courtship elements (Table $2 b$ ) were also recorded for each backcross male and analyzed separately. Variation in courtship intensity was not examined in this study.

\section{Wing dissection and imaging}

The right wings of male backcross progeny were mounted in glycerol with $10 \%$ ethanol on glass slides and pictures of the wings were taken using a Zeiss Axiocam HRC digital camera attached to a Leica MZ7.5 dissecting microscope. All wings were imaged on the same day with the same lighting. Wing spot size was measured in Image $\mathrm{J} 1.31 \mathrm{v}$ by two different workers. Values were divided by wing area (wing length $\times$ wing width) to control for body size effects and then averaged between the two workers.

\section{Results}

\section{Fertility and morphology of $D$. elegans/D. gunungcola $\mathrm{F} 1$} hybrids

D. elegans HK was crossed with D. gunungcola SK in both directions (see Materials and methods). F1 hybrid females from both reciprocal crosses did not differ morphologically from females of either parental strain and were typically fertile, although fertility was not quantified. F1 males from both reciprocal crosses, however, were completely sterile (inferred from their failure to sire progeny in matings with either parental species). Partially or fully deleted abdominal tergites were seen in approximately $50 \%$ of $\mathrm{F} 1$ hybrid males with D. elegans mothers, but F1 males of the reciprocal cross were morphologically normal.

\section{Male wing pigmentation in $\mathrm{F} 1$ hybrids}

Hybrid males with a D. gunungcola mother had clear wings with no apical melanin patch $(N=33)$ (Figure 1d), but hybrid males with a $D$. elegans mother invariably exhibited an apical wing patch, which had a smaller size on average than pure D. elegans males (Figure 1e) (D. elegans/D. gunungcola $\mathrm{F} 1$ males: $N=40$, mean wing spot size corrected for wing size $=0.056 \mathrm{~mm}^{2} / \mathrm{mm}^{2}$; pure $D$. elegans HK males: $N=26$, mean wing spot size corrected for wing size $=0.090 \mathrm{~mm}^{2} / \mathrm{mm}^{2}$ ). The mean spot size difference between D. elegans/D. gunungcola F1 and pure $D$. elegans males was significant (one-tailed t-test, $P<0.0001)$.

Wing spots of D. elegans HK/D. gunungcola SK F1 males were typically less intensely melanized than pure D. elegans HK males (compare Figures $1 \mathrm{~b}$ and $\mathrm{e}$ ), but we did not attempt to quantify this difference. F1 male spots typically had a slightly yellowish tint to their spots, whereas pure D. elegans $\mathrm{HK}$ and other strains tend to be darker brown to black (not shown). 
Genetic analysis of male backcross progeny

F1 female progeny from the cross of D. elegans females to $D$. gunungcola males were used to breed backcross progeny because these were easier to produce than the reciprocal F1 females. A total of 269 male backcross progeny were genotyped at seven loci representing the five major chromosome arms. Wing spot sizes were scored for 125 elegans backcross males (progeny of $D$. elegans $/ D$. gunungcola $F 1$ females $\times D$. elegans $H K$ males) and 99 gunungcola backcross males (progeny of $D$. elegans/D. gunungcola F1 females $\times D$. gunungcola SK males). Courtship was scored for 131 elegans backcross and 95 gunungcola backcross males. Linkage was found only for two pairs of loci, $y$ and Moe $(2.7 \mathrm{cM}$ apart) on the $\mathrm{X}$-chromosome and $e$ and TfIIA-L $(7.5 \mathrm{cM}$ apart) on the presumptive third-chromosome right arm (3R). This linkage is as expected from the D. melanogaster genome and suggests that the relative positions of these genes are similar to their homologs in D. melanogaster. However, these recombination rates are much lower than expected from D. melanogaster (27.5 and $21.8 \mathrm{cM}$, respectively).

Wing spot size in backcross populations

Overall, the wing spot size of backcross males was continuously distributed, varying from no spot to a spot very close to pure $D$. elegans in size. The two backcross populations differed significantly in mean wing spot size (Mann-Whitney $U$-test, $P<0.0001$ ). The predominant effect of the $D$. elegans X-chromosome was apparent; around half of all backcross males lack wing spots (see below). This corresponds to inheritance of an intact or nearly intact $D$. gunungcola X-chromosome. The continuous distribution of wing spot size among males possessing wing spots suggests that the species difference is polygenic, involving at least one autosomalmodifying locus in addition to one or more loci of collectively large effect on the X-chromosome.

Mean spot sizes for each recovered genotypic class in the backcross populations are shown in Figure 2. In the elegans backcross population (Figure 2a), only three genotypic classes lacking the $D$. elegans $\mathrm{X}$-chromosome, marked by genotype at the $y$ locus, possessed wing spots of any size, and all three of these classes carried the $D$. elegans homolog of the D. melanogaster right arm of the chromosome III (3R), marked by $e$. However, carrying the D. elegans homolog of $3 R$ was not sufficient to confer a wing spot as several genotypic classes carrying this element did not exhibit wing spots. In the gunungcola backcross population (Figure 2b), no genotypes lacking the D. elegans X-chromosome exhibited wing spots. Overall, wing spots in the gunungcola

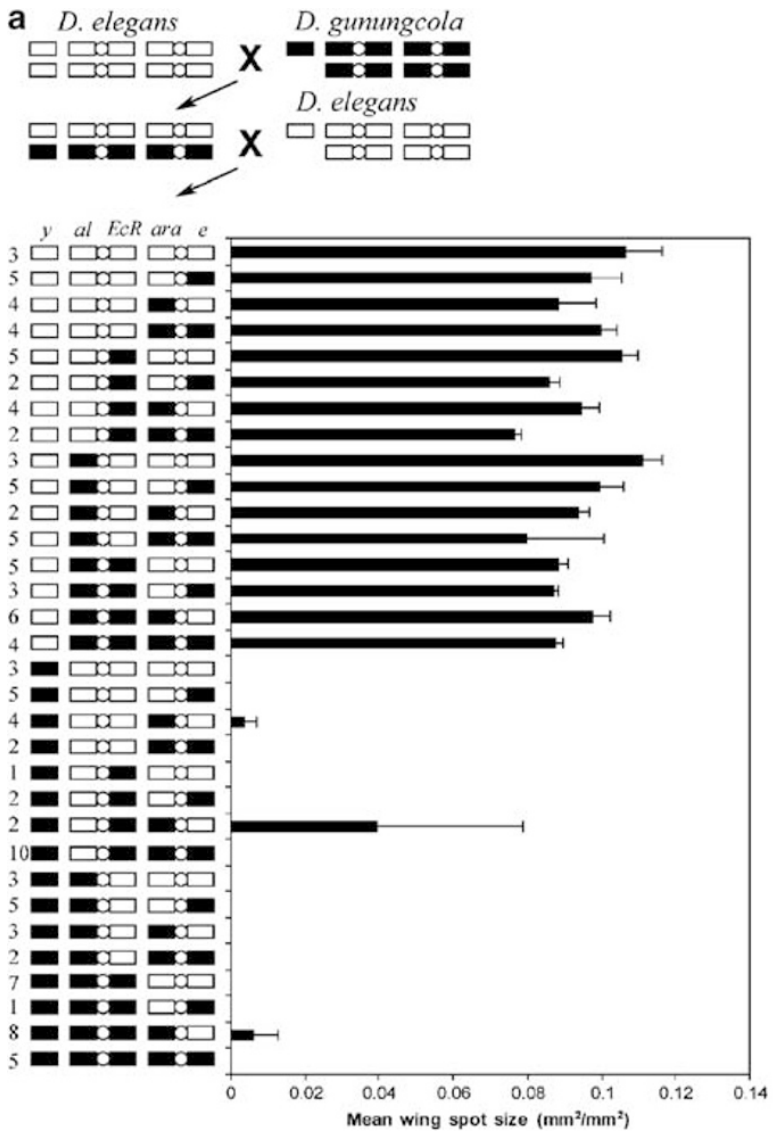

b
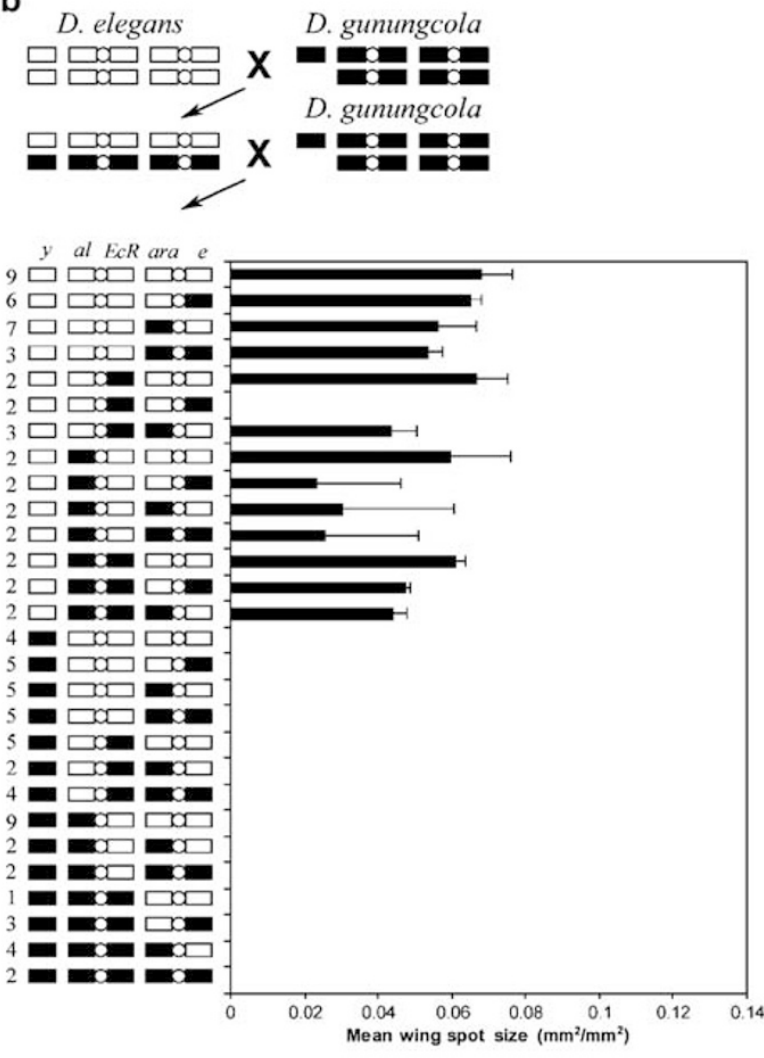

Figure 2 Genetic analysis of wing spot size in elegans and gunungcola backcross populations. (a) elegans backcross males. (b) gunungcola backcross males. Backcross is shown at the top of each panel. White and black rectangles represent marked D. elegans and D. gunungcola genome segments, respectively. $X$, left and right arms of second chromosome, and left and right arms of third chromosome, from left to right, are indicated ( $\mathrm{Y}$ and fourth chromosomes are not shown and were not analyzed in this study). For backcross progeny at the left, the invariable haplotype inherited from the male backcross parent is not shown. Numbers on left indicate sample sizes of each genotype. Error bars represent 1 SE. Genotypes with no error bars had either no variance or sample size of one. Some genotypes were not present in the backcross populations. The $\mathrm{X}$ is represented by $y$ and the presumptive third chromosome right arm (3R) is represented by $e$. 
backcross population were smaller on average than those in the elegans backcross population.

In the F1 hybrid background, the D. elegans Xchromosome was necessary for the production of wing spots. The $D$. elegans backcross data (Figure 2a) are largely consistent with the necessity of the D. elegans Xchromosome for spot production. Among individuals bearing wing spots and carrying the D. gunungcola allele at the X-linked $y$ marker, a total of four individuals also carried the D. gunungcola allele at the other X-linked marker, Moe. Nevertheless, because these two markers may not cover the entire X-chromosome (they span only about $42 \%$ of the $\mathrm{X}$-chromosome in D. melanogaster), we cannot rule out the possibility that these individuals were recombinants that still possessed some part of the D. elegans X-chromosome. In the gunungcola backcross population (Figure $2 \mathrm{~b}$ ), the $D$. elegans X-chromosome, or at least that portion linked to $y$ and Moe, appeared to be necessary for the production of a wing spot. However, one genotypic class with the D. elegans $y$ allele, represented by two individuals, had no wing spots. One of these two individuals also had the $D$. elegans allele at Moe and the other had the D. gunungcola Moe allele.

The potential sufficiency of the D. elegans X-chromosome for spot production could in principle be addressed by examining the $D$. gunungcola backcross population. Two individuals from this backcross possessed the $D$. elegans allele at both X-linked markers and yet had no wing spots (not shown). However, one of these two individuals carried the D. elegans allele at the al and ara markers. The other carried the D. elegans allele at all of the autosomal marker loci, except ara. We also cannot rule out possession by these nonspot-bearing males of some fraction of the D. elegans X-chromosome outside the y-Moe interval.

\section{Marker association tests for wing spot size}

Two types of tests were used to determine whether marker genotypes were associated with wing spot size in the backcross populations (Figure 3). A single marker association analysis was performed by using a $t$-test to test the hypothesis of equal means of different genotypes at the same locus, pooled irrespective of genotypes at the other marker loci. Also, a factor analysis was performed by analysis of variance (ANOVA) with each of the seven markers as an effect in the model. The results of these two tests were fairly similar. In both backcross populations, $y$ was significantly associated with wing spot size in both tests but Moe showed a significant effect on wing spot size only in the single marker test. Since $y$ and Moe are on the X-chromosome, this might suggest that the $\mathrm{X}$ linked locus (or loci) responsible for wing spot are closer to $y$ than to Moe, but the power of the ANOVA test is low due to small sample sizes for each genotypic class. The presumptive 2L marker al showed a significant effect in wing spot size, but only in the gunungcola backcross (Figure $3 b$ ), which implies dominance of $D$. elegans allele(s) affecting wing spot size on $2 \mathrm{~L}$. $e$ showed a significant effect in the ANOVA test in the elegans (Figure 3a), but not the gunungcola (Figure 3b) backcross
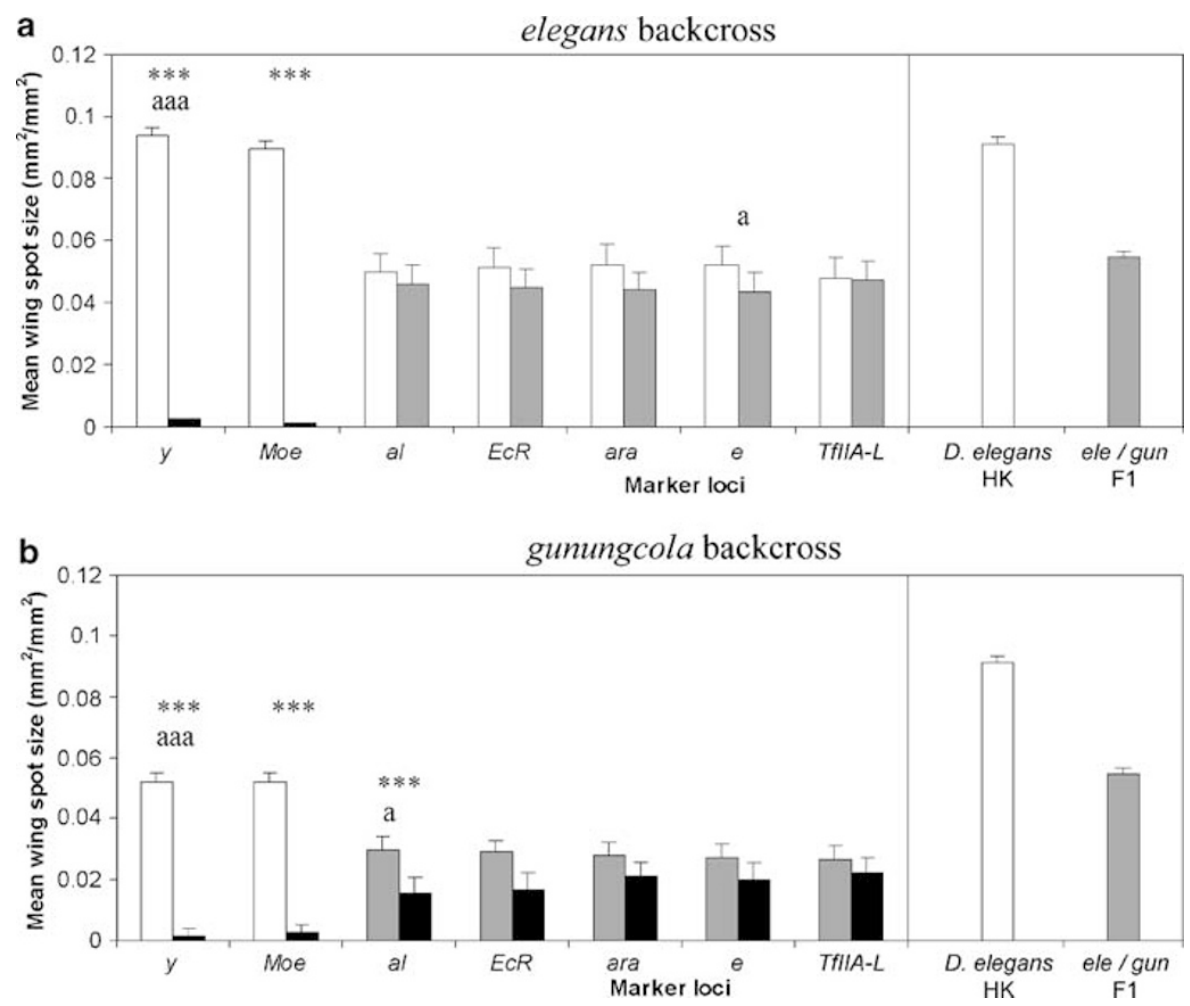

Figure 3 Wing spot size of genotypic classes at each marker locus. (a) elegans backcross males. (b) gunungcola backcross males. White bars are hemizygous or homozygous for elegans alleles. Black bars are hemizygous or homozygous for gunungcola alleles. Gray bars are heterozygous for elegans and gunungcola alleles. For $t$-test on one marker, ${ }^{* * *} P<0.0001$. For ANOVA test, aaa $P<0.0001$; ${ }^{a} P<0.05$. Error bars indicate $1 \mathrm{SE}$. On the right of both panels, mean spot sizes of pure D. elegans HK and D. elegans/D. gunungcola $\mathrm{F} 1$ hybrids (with D. elegans X-chromosome) are shown. 
population, suggesting the presence of a dominant D. gunungcola allele reducing wing spot size.

\section{Courtship of $D$. elegans}

D. elegans male courtship begins with orientation of the male toward the female, followed by tapping of the female abdomen with his forelegs. Then, if the male maintains interest, he circles from the back of the female to her front, facing her head, with one wing extended. While circling, the male's head faces the female and the wing on the side closest to the female head is fully extended and maintained in extended position. After he moves to her head, the male shakes his body with both wings opened, which is referred to as the wing display. While shaking his body, the male opens and steadily holds both wings at approximately $180^{\circ}$ angle (perpendicular to the anterior-posterior axis of his body), while turning the ventral surfaces of the wings forward, lifting his head and thorax, keeping his abdomen close to the substrate or bending the tip of his abdomen toward the female. (Body shaking can also occur without wing extension, but we have only seen this in backcross progeny of $D$. elegans/D. gunungcola hybrids.) During a bout of courtship, the male typically tries to stay directly in front of the female while displaying, often adjusting his position to maintain this orientation in response to female movement. After one or more wing displays, the male moves behind the female, bends the tip of his abdomen toward the female terminalia, grasps her posterior abdomen with his forelegs, and attempts to copulate with her. When he is attempting to copulate, one of his wings is typically extended. If the copulation attempt is not successful, the male may remain behind the female with one wing extended for several seconds and then attempt to copulate again. Alternatively, he may repeat circling and frontal wing display.

\section{Courtship of $D$. gunungcola}

Like $D$. elegans, $D$. gunungcola males also tap the female abdomen with their forelegs before courting the female. Courtship consists of the male extending one or both wings to about a $30^{\circ}$ angle to his body and then back over his abdomen repeatedly and rapidly. Then, the male attempts to copulate with the female. Unlike D. elegans males, D. gunungcola males remain behind or to the side of the females during courtship. They do not circle to the front of the females, and they do not hold their wings perpendicular to their body at any time.

Courtship of $D$. elegans/D. gunungcola $\mathrm{F} 1$ hybrid males Hybrid males from both reciprocal crosses showed wing display and male circling in their courtship. However, the courtships of hybrid males from the reciprocal crosses were not identical, and neither F1 genotype was the same as pure D. elegans males. Shaking of the body during the frontal wing display was much less pronounced than pure D. elegans and often the wings were not held fully out during the display. Furthermore, in F1 males with $D$. gunungcola mothers $(N=33)$, wings were usually held out one by one, not simultaneously, during the frontal display. F1 males with $D$. elegans mothers $(N=40)$ usually held their wings out simultaneously during the frontal display, but this was seen less frequently in F1 males from the reciprocal cross. The hybrid males from both reciprocal crosses showed less frequent wing display than pure $D$. elegans males. Courtship of both reciprocal F1 male genotypes can be classified as category 3 in our numerical classification system (see below and Table 2a).

\section{Courtship in backcross populations}

Distributions of courtship scores in the elegans and gunungcola backcross populations are shown in Figure 4. In both backcrosses, a continuous distribution of scores was seen, with the elegans backcross males showing higher scores than the gunungcola backcross males, as expected from the parental species differences. The two backcross populations differed significantly in mean courtship score (Mann-Whitney $U$-test, $P<0.0001$ ).

The mean courtship scores of genotypic classes are depicted for the elegans backcross population in Figure 5a and the gunungcola backcross population in Figure 5b. The consistently higher scores in the elegans backcross population compared to the gunungcola backcross population indicate that the autosomal backgrounds differ at one or more loci affecting the courtship difference between species. The X-chromosome appeared to have a subtle effect in the elegans backcross population (cf. genotypes carrying and lacking the elegans X-chromosome in Figure 5a and see below).

\section{Marker association tests for courtship score}

As with the wing spot data, single marker association and factor analysis (ANOVA) tests were was performed on the courtship data. Results are shown in Figure 6. In both backcrosses, $y$ and Moe showed significant associations with courtship in the single marker analysis (but not in the ANOVA), suggesting the existence of an Xlinked locus or loci affecting the wing display difference between species. Few other markers showed strong effects on courtship score, although most of the markers showed significant effects in the gunungcola backcross (Figure 6b).

\section{Genetics of divergence in courtship elements}

The genetic basis of courtship divergence between $D$. elegans and D. gunungcola was studied in more detail by

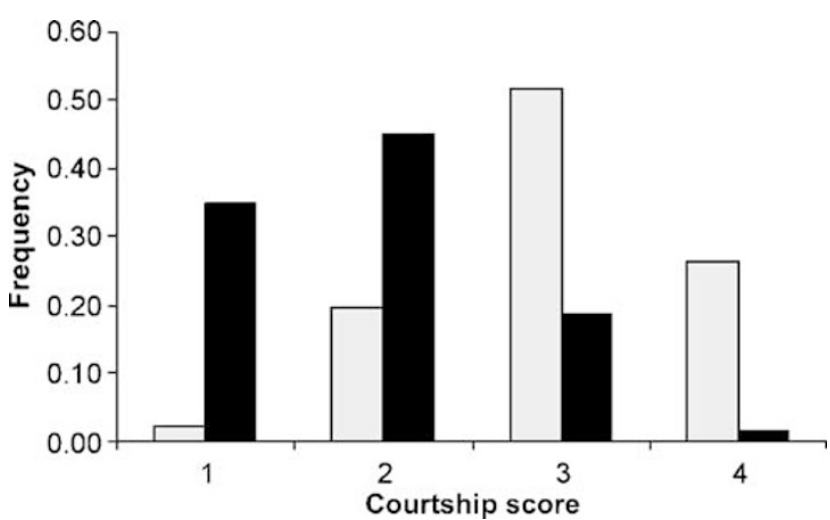

Figure 4 Distribution of male courtship score in backcross populations. White bars indicate elegans backcross progeny. Black bars represent gunungcola backcross progeny. Pure D. elegans males have a courtship score of 4 . Pure D. gunungcola males have a courtship score of 1. F1 males of both reciprocal genotypes have courtship scores of 3 (see text). 

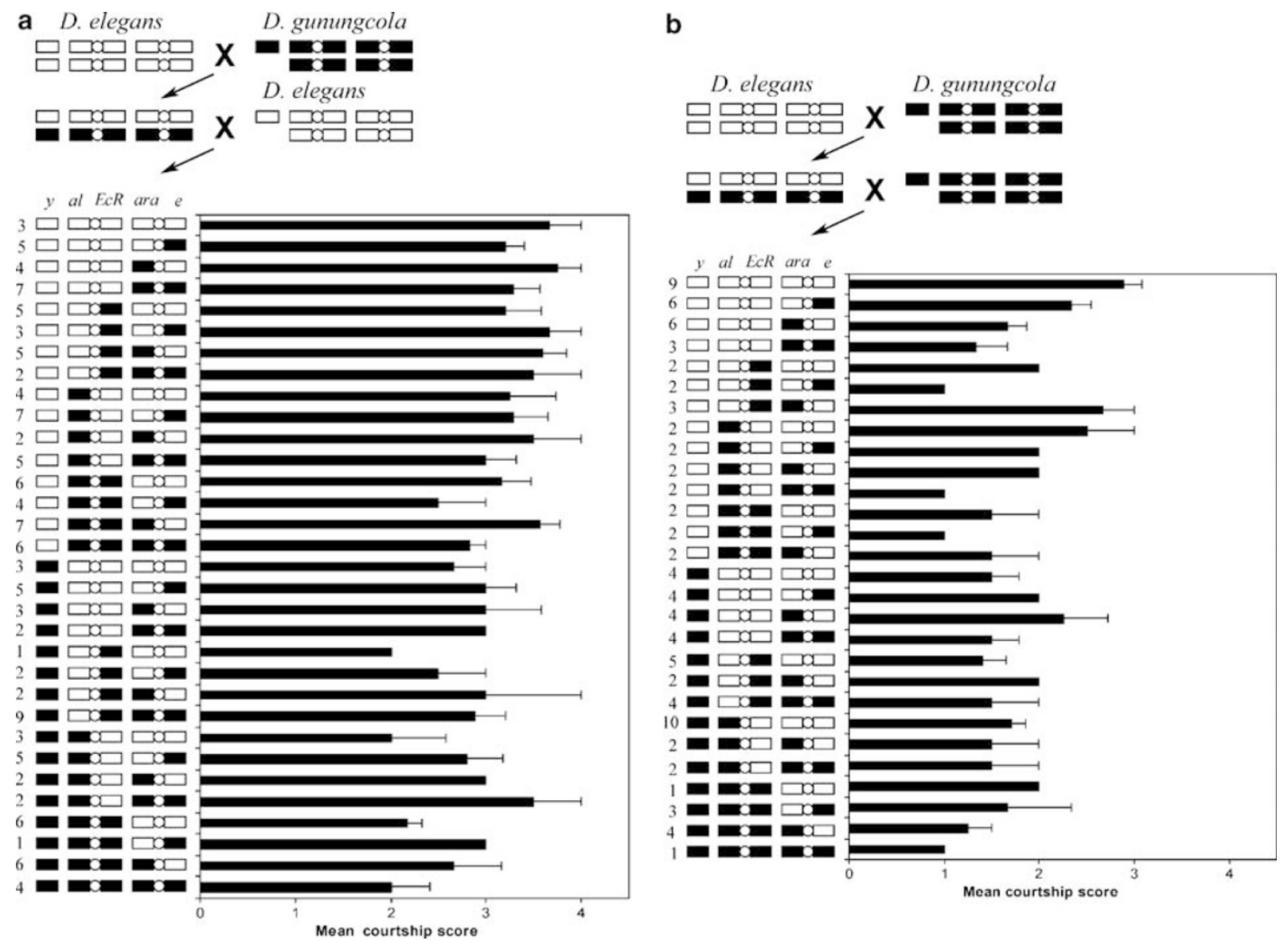

Figure 5 Genetic analysis of male courtship score in elegans and gunungcola backcross populations. (a) elegans backcross males. (b) gunungcola backcross males. Backcross is shown at the top of each panel. White and black rectangles represent marked D. elegans and D. gunungcola genome segments, respectively. $X$, left and right arms of second chromosome, and left and right arms of third chromosome, from left to right, are indicated ( $\mathrm{Y}$ and fourth chromosomes are not shown and were not analyzed in this study). For backcross progeny at the left, the invariable haplotype inherited from the male backcross parent is not shown. Numbers on the left indicate sample sizes of each genotype. Error bars represent 1SE. Genotypes with no error bars had either no variance or sample size of one. Some genotypes were not present in the backcross populations. The $\mathrm{X}$ is represented by $y$ and the presumptive third chromosome right arm (3R) is represented by $e$.

examining the presence or absence of three $D$. elegansspecific elements (Table $2 b$ ) in the backcross progeny. These were the presence or absence of a display utilizing both wings (wing display), circling, and body shaking.

Results of marker association tests for each of these elements are shown in Table 3. Interestingly, the different courtship elements showed evidence of separable genetic architectures controlling the species differences. The two backcross populations gave different results, suggesting dominance of some of the loci underlying species differences. In the elegans backcross population (Table 3, top), wing display showed significant influences of $X$ chromosome markers. These associations were not seen in the gunungcola backcross population (Table 3, bottom), suggesting that $D$. elegans alleles at these loci are recessive to $D$. gunungcola alleles. In the gunungcola backcross population, al showed a significant association with the wing display element. This suggests that a $D$. elegans allele in the vicinity of this marker has a dominant effect on the species difference in wing display. Only markers on the second chromosome were associated with circling behavior, with al showing association in the elegans backcross and $E c R$ showing an association in the gunungcola backcross. Finally, body shaking showed associations with the linked markers $e$ and TfIIA-L in both backcrosses with a significant effect of $E c R$ in the gunungcola backcross.

In the entire analysis of courtship elements, 42 tests were performed. By chance, 2.1 tests were predicted to show significance at the 5\% level. Our analysis showed 11 such significant results, six with $P$-values less than 0.01 . This suggests that at least some of these marker associations were real and not random artifacts in the data.

Correlations among male traits in backcross progeny In both backcross populations, male wing spot size was highly significantly correlated with courtship score (elegans backcross: Kendall's $\tau=0.242, \quad P<0.0001$; gunungcola backcross: $\tau=0.348, P<0.0001)$. These correlations may be due either to linkage of loci responsible for these two traits or low recombination rates in F1 hybrid females (see above). Alternatively, one or more genetic factors may be pleiotropic, affecting more than one of the traits. 
a elegans backcross
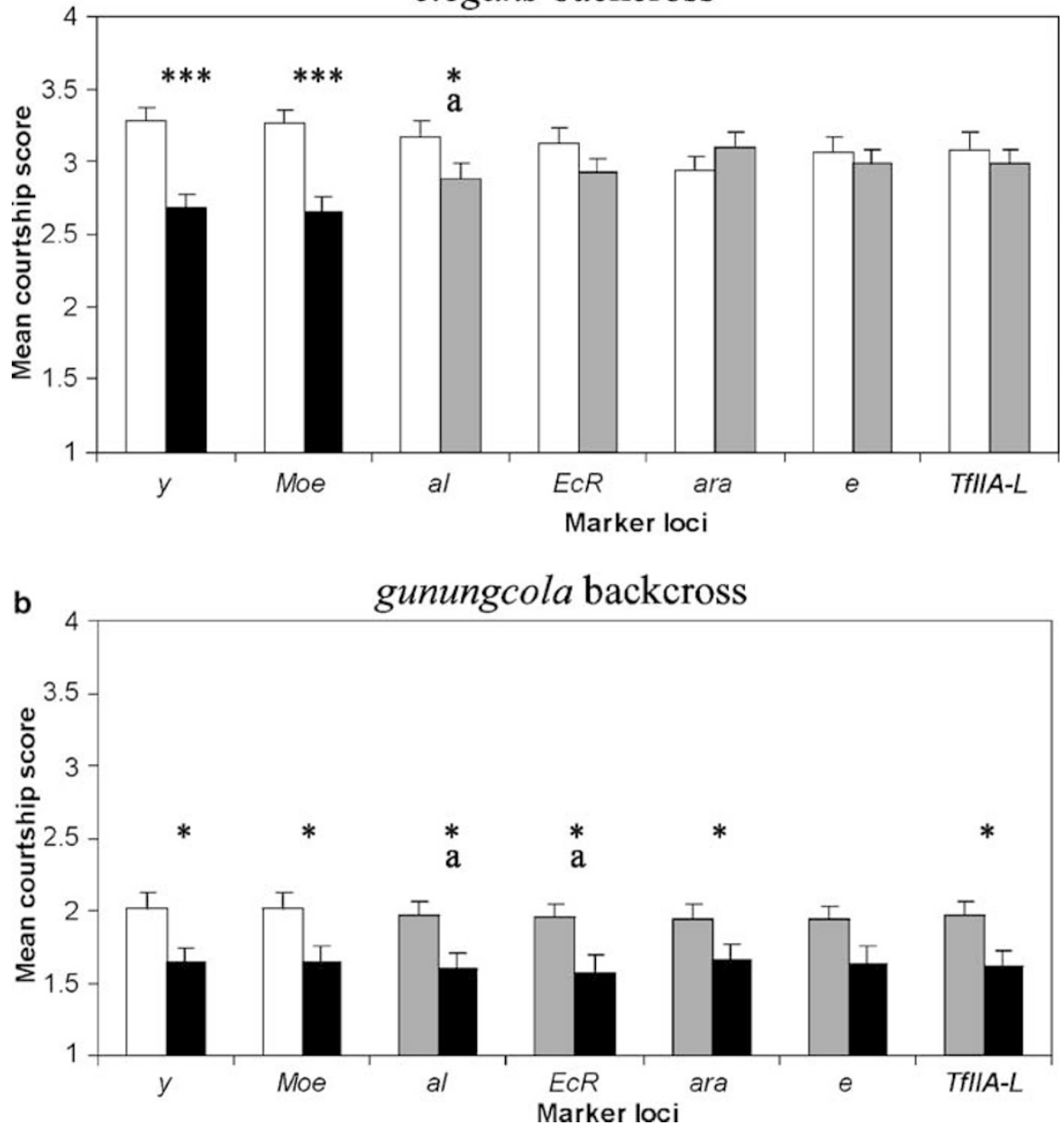

Figure 6 Courtship score of genotypic classes at each marker locus. (a) elegans backcross males. (b) gunungcola backcross males. White bars are hemizygous or homozygous for elegans alleles. Black bars are hemizygous or homozygous for gunungcola alleles. Gray bars are heterozygous for elegans and gunungcola alleles. For $t$-test on one marker, ${ }^{* * *} P<0.0001 ;{ }^{*} P<0.05$. For ANOVA test, a $P<0.05$. Error bars indicate 1SE.

\section{Discussion}

This study represents an initial, low-resolution analysis of the genetic architecture of divergent male pigmentation and courtship behavior between $D$. elegans and $D$. gunungcola. Before discussing the biological significance of our findings, we must first mention several caveats to this analysis. First, the genetic resolution is very low. Only one marker or one linked pair of markers was used per chromosome arm and the fourth chromosome ( $2 \%$ of the genome in D. melanogaster) was not studied. Moreover, the positions of genetic factors affecting species differences within chromosomes and the magnitudes of their effects cannot be estimated with the current data. Although the number of chromosome arm elements is conserved throughout the $D$. melanogaster species group (Ashburner, 1989), we cannot be sure at this time that all chromosome arms were represented in this study, since genomic rearrangements, including translocations, may have taken place since the common ancestor of $D$. melanogaster and D. elegans/D. gunungcola. Nevertheless, our markers do not show evidence of linkage other than between the linked pair $y$ and Moe, both of which are known to be on the D. elegans X-chromosome, and the tightly linked pair $e$ and TfIIA-L, which are known to be linked on $3 \mathrm{R}$ in $D$. melanogaster. Thus, the genomic coverage provided by our markers is the same as one marker per chromosome arm.

Another caveat involves the simplification of male courtship behavior into an ordinal score. This score doubtless misses many subtleties of courtship in the backcross hybrid males. However, we believe this scoring system captured a substantial amount of the variation in the backcross populations. Our analysis of individual courtship elements was consistent with the results from the ordinal scoring system.

\section{Large X-effect on male wing pigmentation}

Both male wing spots and courtship behavior have polygenic architectures controlling the species differences between $D$. elegans and D. gunungcola. Most strikingly, the $D$. elegans $X$-chromosome appears to confer a very large effect on wing spot size. The presence of at least two factors promoting wing spot formation/size is suggested by the approximately continuous distribution 
Table 3 Effects of marker genotype on divergent male courtship elements scored in backcross progeny

\begin{tabular}{lccc}
\hline Marker (arm) & Wing display & Circling & Body shaking \\
\hline Elegans backcross & population & & \\
y $(\mathrm{X})$ & 0.0110 & $\mathrm{NS}$ & $\mathrm{NS}$ \\
Moe $(\mathrm{X})$ & 0.0070 & $\mathrm{NS}$ & $\mathrm{M}$ \\
al $(2 \mathrm{~L})$ & $\mathrm{NS}$ & 0.0015 & $\mathrm{NS}$ \\
EcR $(2 \mathrm{R})$ & $\mathrm{M}$ & $\mathrm{NS}$ & $\mathrm{NS}$ \\
ara $(3 \mathrm{~L})$ & $\mathrm{NS}$ & $\mathrm{NS}$ & $\mathrm{NS}$ \\
e $(3 \mathrm{R})$ & $\mathrm{NS}$ & $\mathrm{NS}$ & 0.0167 \\
TfIIA (3R) & $\mathrm{NS}$ & $\mathrm{NS}$ & 0.0055 \\
& & & \\
Gunungcola backcross & population & & \\
$y(X)$ & $\mathrm{NS}$ & $\mathrm{NS}$ & 0.0239 \\
Moe $(X)$ & $\mathrm{NS}$ & $\mathrm{NS}$ & $\mathrm{NS}$ \\
al $(2 L)$ & 0.0199 & $\mathrm{NS}$ & $\mathrm{NS}$ \\
EcR $(2 R)$ & $\mathrm{NS}$ & 0.0032 & 0.0280 \\
ara $(3 L)$ & $\mathrm{NS}$ & $\mathrm{NS}$ & $\mathrm{NS}$ \\
e $(3 R)$ & $\mathrm{NS}$ & $\mathrm{NS}$ & 0.0059 \\
TfIIA $(3 R)$ & $\mathrm{M}$ & $\mathrm{M}$ & 0.0041 \\
\hline
\end{tabular}

Arm refers to chromosome arm in D. melanogaster. Arm location has been confirmed for X-linked markers in D. elegans and $D$. gunungcola, but not autosomal markers. Numbers indicate $P$-values of nominal logistic regressions in JMP.

Significant $P$-values are indicated. NS: not significant. M: marginal significance; $0.10>P>0.05$.

of wing spot sizes in the backcross progeny. F1 hybrid males carrying the $D$. elegans X-chromosome had, on average, wing spots that were about $62 \%$ the size of pure D. elegans spots and the F1 spots are typically not as dark as pure $D$. elegans spots. This indicates that one or more genes in the $D$. elegans autosomal background with apparently smaller effects than the X-linked gene(s) are also required for the production of spots with full size and intensity.

Large X-effects have been found in the genetic basis of postzygotic reproductive isolation in many Drosophila species pairs (Coyne and Orr, 1989), the most thoroughly studied of which is D. simulans/D. mauritiana (Hollocher and $\mathrm{Wu}, 1996$; True et al, 1996). This is potentially related to a larger pattern among animals in which intra- and interspecific differences in sex-specific characters tend to show large X-effects in their genetic architecture (Prowell, 1998; Reinhold, 1998; Paallysaho et al, 2003). These effects are also consistent with recent applications of sexual selection theory, including Fisher's runaway and 'good genes' models (Kirkpatrick and Hall, 2004a, b). Large X-effects on sexually selected traits are also predicted consequences of sexual antagonism by genes selectively favored in one sex but disadvantageous in the other (Rice, 1984), the 'dominance' theory of hybrid incompatibility genes (reviewed by Turelli and Orr, 2000), and the greater exposure of X-linked recessive alleles to selection in males in comparison with autosomal recessive alleles (Charlesworth et al, 1987). However, theoretical work by Reeve and Pfennig (2003) argued that large $\mathrm{X}$-effects (Z-effects) are more likely to underlie divergence in male secondary sexual traits in male homogametic species than female homogametic species such as Drosophila. It should also be pointed out that the X-chromosome constitutes roughly one-fifth of the genome in the melanogaster species group, a large proportion relative to other Drosophila lineages (Patterson and Stone, 1952; Ashburner, 1989), so on the basis of chance alone large X-effects underlying D. elegans/ gunungcola trait differences might not be too surprising.

We have found that one potential component of prezygotic reproductive isolation, male wing spots, exhibits a strong X-effect, but that another component, male courtship, does not. Large X-effects in traits involved in sexual isolation are far from universal. Many studies on various traits involved in male courtship, especially courtship song, have failed to find evidence for large X-chromosome effects (Cowling and Burnet, 1981; Kawanishi and Watanabe, 1981; Kyriacou and Hall, 1986; Tomaru and Oguma, 1994; Pugh and Ritchie, 1996; Noor, 1997; Colegrave et al, 2000; Gleason et al, 2002; Gleason and Ritchie, 2004; Huttunen et al, 2004; Moehring and Mackay, 2004). In the present case, the explanation for the large X-effect on male wing spots could be that the most important variable pigmentation genes, possibly $y$ and tan, happen to reside on the $X$-chromosome. Therefore, the role of the $\mathrm{X}$-chromosome in species divergence could depend critically on genomic history. This would predict that species closely related to $D$. elegans and D. gunungcola should also show strong Xeffects on pigmentation differences between species, but that this may not apply to distantly related lineages.

Modern adaptation theory (Kimura, 1983; Orr, 1998, 2001, 2005) posits that selection toward a phenotypic optimum should involve early large steps and later steps of progressively smaller effect ('fine-tuning' or 'modifying' factors). In the case of male-specific traits that are the product of sexual selection, such modifiers may be favored by stabilizing sexual selection if a particular (ie intermediate) size of the male ornament is favored, as opposed to an ever increasing ornament size that would be favored by runaway sexual selection. Thus, one possible trajectory for the evolution (or loss) of male wing spots may have been to first substitute alleles of major effect at the X-linked loci, followed by accumulation of modifier alleles at the autosomal loci.

\section{Candidate genes for melanin pattern evolution}

Two important candidate genes for melanin pattern evolution reside on the $X$-chromosome, $y$ and $t$. In $D$. melanogaster, $y$ is required for dopa melanization; $y$ hypomorphic or null individuals have light yellowish brown pigment instead of dark black in their cuticle and bristles (Lindsley and Zimm, 1992). Yellow is also expressed in the male-specific spot region in late pupal D. elegans and other spot-bearing melanogaster group species (Kopp and True, 2002a; Wittkopp et al, 2002; Gompel et al, 2005). Yellow is an extracellular protein thought to function specifically in the dopa melanin pathway, either by sequestering secreted dopa or by functioning in one of the enzymatic steps during oxidative polymerization of dopa to melanin (Walter et al, 1991; Wittkopp et al, 2002). Ectopic y expression promotes novel melanin pattern formation in $e$ mutant backgrounds (Wittkopp et al, 2002), which is one possible mechanism by which novel melanin patterns could evolve (True, 2003). Interestingly, Drapeau et al (2003) recently demonstrated that male-specific $y$ expression in the brain is required for normal wing extension during male courtship. Such pleiotropic effects could play a significant role in the coordinate evolution of malespecific pigmentation and behavior. 
The tan gene encodes $N$ - $\beta$-alanyl dopamine (NBAD) hydrolase, which is required to provide dopamine for melanization, by converting sequestered NBAD (produced by Ebony) to dopamine in epidermal cells (Wright, 1987; True et al, 2005). Ectopic tan expression is sufficient to promote novel melanin pattern formation (True et al, 2005), but its expression pattern has not yet been established. Spatially regulated gains and losses of tan expression could underlie the wing and body pigmentation differences between $D$. elegans and $D$. gunungcola.

Our marker association study suggests that wing spot size is affected by one or more factors in the vicinity of the $e$ locus in the elegans backcross population and the al locus in the gunungcola backcross population. The differential results between the two backcrosses suggest that these factors may possess differential dominance; the $e$-linked $D$. elegans factor appears to be recessive, while the al-linked $D$. elegans factor appears to be dominant. QTL mapping with more genetic markers is needed to resolve the location and number of these autosomal effects on wing spot size. One interesting autosomal candidate gene for wing spot size is $e$ itself. $e$ is expressed in the nonmelanized areas of wings and bodies in several melanogaster group species (Wittkopp et al, 2002; Gompel et al, 2005), where it may be necessary to inhibit melanization.

Genetic basis of courtship differences between species Our analysis of the genetics of male courtship divergence is one of the first attempts to genetically dissect a discrete courtship difference between closely related species. Intermediate behavioral phenotypes are common in species hybrids and have been found for a variety of traits, including migratory behavior in birds (Helbig, 1991) and courtship behavior in birds (Ficken and Ficken, 1968) and spiders (Stratton and Uetz, 1986). Pioneering work by Shaw $(1996,2000)$ characterized the quantitative genetic basis of differences in male courtship song and female preferences among species in the cricket genus Laupala. Both traits were found to be polygenic, suggestive of gradual, incremental divergence (Shaw, 2000). By contrast, Doi et al (2001) mapped a single locus or linked gene complex near the autosomal gene Delta that largely controls female preferences for male courtship song in the D. ananassae/D. pallidosa species pair.

The observation that reciprocal F1 hybrid males differ only subtly in their courtship suggests that the major loci controlling courtship differences are on the autosomes. However, evidence of one or more X-linked factors is provided by the backcross progeny analysis. Intralocus dominance and differential genetic background effects are apparent, as most of the markers had effects in only one of the two backcross populations. Another intriguing finding was that the genetic architectures of specific courtship elements that differ between the species appear to be at least partially separable. In particular, wing display appeared to be largely influenced by Xchromosomal genotypes, whereas body shaking was more strongly dependent on third chromosome genotypes. The only marker effects on circling behavior were found for second chromosome markers. Such a genetically separable pattern of elements composing a complex behavioral trait suggests that the more complex court- ship in D. elegans, or its loss in D. gunungcola, may have occurred by a stepwise or incremental accumulation of elements involving movement of distinct anatomical parts.

\section{Correlations among male wing spots and courtship behavior}

Although the genetics of the male wing spot and display can be somewhat separated, as evidenced by reciprocal F1 male phenotypes, nevertheless we have also found evidence of overlaps in the genetic architectures of wing spot and courtship divergence between D. elegans and D. gunungcola. In particular, the X-chromosome largely determines the presence or absence of wing spots and also has highly significant effects in the backcross populations on wing display.

More detailed QTL analysis is needed to resolve whether linked or possibly pleiotropic loci underlie divergence in pigmentation and behavior in this species pair. Close physical linkage of pigmentation and behavior genes with major effects could constrain trajectories of short-term evolution by producing persistent linkage disequilibrium between alleles conferring particular pigmentation and behavioral states. Single loci underlying variation in pigmentation and behavioral traits could similarly bias the types of pigmentation-behavior correlations that evolve, depending on the degree of independence of their regulation.

\section{Acknowledgements}

We thank MT Kimura for kindly providing D. elegans and D. gunungcola stocks. M Bello and S Harry provided excellent technical assistance. A Kopp and G Gibson provided valuable advice on molecular marker techniques. R Geeta, J LaChance, J Wiens, D Futuyma, $\mathrm{R}$ Yukilevich, and two anonymous reviewers provided many helpful comments during the writing and revision of this manuscript. This work was supported by funds from SUNY Stony Brook. DNA sequencing was performed in the National Science Foundation-funded MEAD Laboratory in the SUNY Stony Brook Department of Ecology and Evolution. This paper is number 1143 contributed by the Graduate Program of the Department of Ecology and Evolution at SUNY Stony Brook.

\section{References}

Andersson M (1994). Sexual Selection. Princeton University Press: Princeton, NJ.

Ashburner M (1989). Drosophila: A Laboratory Handbook. Cold Spring Harbor Press: Cold Spring Harbor, NY.

Charlesworth B, Coyne JA, Barton NH (1987). The relative rates of evolution of sex chromosomes and autosomes. Am Nat 130: 113-146.

Colegrave N, Hollocher H, Hinton K, Ritchie MG (2000). The courtship song of African Drosophila melanogaster. J Evol Biol 13: 143-150.

Cowling DE, Burnet B (1981). Courtship songs and genetic control of their acoustic characteristics in sibling species of the Drosophila melanogaster subgroup. Anim Behav 29: 924-935.

Coyne JA, Orr HA (1989). Two rules of speciation. In: Otte D, Endler JA (eds) Speciation and its Consequences. Sinauer Associates: Sunderland, MA. pp 180-207. 
Doi M, Matsuda M, Tomaru M, Matsubayashi H, Oguma Y (2001). A locus for female discrimination behavior causing sexual isolation in Drosophila. Proc Natl Acad Sci USA 98: 6714-6719.

Drapeau MD, Radovic A, Wittkopp PJ, Long AD (2003). A gene necessary for normal male courtship, yellow, acts downstream of fruitless in the Drosophila melanogaster larval brain. J Neurobiol 55: 53-72.

Ficken MS, Ficken RW (1968). Courtship of blue-winged warblers, golden-winged warblers, and their hybrids. Wilson Bull 80: 161-172.

Gleason JM, Nuzhdin SV, Ritchie MG (2002). Quantitative trait loci affecting a courtship signal in Drosophila melanogaster. Heredity 89: 1-6.

Gleason JM, Ritchie MG (2004). Do quantitative trait loci (QTL) for a courtship song difference between Drosophila simulans and $D$. sechellia coincide with candidate genes and intraspecific QTL? Genetics 166: 1303-1311.

Gompel N, Prud'homme B, Wittkopp PJ, Kassner VA, Carroll SB (2005). Chance caught on the wing: cis-regulatory evolution and the origin of pigment patterns in Drosophila. Nature 433: 481-487.

Greene E, Orsak LJ, Whitman DW (1987). A tephritid fly mimics the territorial displays of its jumping spider predators. Science 236: 310-312.

Greenspan RJ, Ferveur JF (2000). Courtship in Drosophila. Annu Rev Genet 34: 205-232.

Hawthorne DJ, Via S (2001). Genetic linkage of ecological specialization and reproductive isolation in pea aphids Nature 412: 904-907.

Helbig AJ (1991). Inheritance of migratory direction in a bird species: a cross-breeding experiment with SE- and SWmigrating blackcaps (Sylvia atricapilla). Behav Ecol Sociobiol $\mathbf{2 8}$ 9-12.

Higashi M, Takimoto G, Yamamura N (1999). Sympatric speciation by sexual selection. Nature 402: 523-526.

Hirai Y, Kimura MT (1997). Incipient reproductive isolation between two morphs of Drosophila elegans (Diptera: Drosophilidae). Biol J Linn Soc 61: 501-513.

Hollocher H, Wu C-I (1996). The genetics of reproductive isolation in the Drosophila simulans clade: $\mathrm{X}$ vs autosomal effects and male $v$ s female effects. Genetics 143: 12431255.

Huttunen S, Aspi J, Hoikkala A, Schlotterer C (2004). QTL analysis of variation in male courtship song characters in Drosophila virilis. Heredity 92: 263-269.

Ishii K, Hirai Y, Katagiri C, Kimura MT (2002). Mate discrimination and cuticular hydrocarbons in Drosophila elegans and D. gunungcola. Zool Sci 19: 1191-1196.

Kaneshiro KY, Boake CRB (1987). Sexual selection and speciation: issues raised by Hawaiian Drosophila. Trends Ecol Evol 2: 207-212.

Kaneshiro KY (2000). Sexual selection and speciation in Hawaiian Drosophila (Drosophilidae): A model system for research in Tephritidae. In: Aluja M, Norrbom AL (eds) Fruit Flies (Tephritidae): Phylogeny and Evolution of Behavior. CRC Press: New York. pp 861-877.

Kawanishi M, Watanabe TK (1981). Genes affecting courtship song and mating preference in Drosophila melanogaster, Drosophila simulans and their hybrids. Evolution 35: 11281133.

Kimura M (1983). The Neutral Theory of Molecular Evolution. Cambridge University Press: Cambridge, UK

Kimura MT, Hirai Y (2001). Daily activity and territoriality of Drosophila elegans in Sukarami, West Sumatra, Indonesia. Tropics 10: 489-495.

Kirkpatrick M, Hall DW (2004a). Male-biased mutation, sex linkage, and the rate of adaptive evolution. Evolution 58: 437-440.

Kirkpatrick M, Hall DW (2004b). Sexual selection and sex linkage. Evolution 58: 683-691.
Kopp A, True JR (2002a). Evolution of male sexual characters in the Oriental Drosophila melanogaster species group. Evol Devel 4: 278-291.

Kopp A, True JR (2002b). Phylogeny of the Oriental Drosophila melanogaster species group: a multi-locus reconstruction. Syst Biol 51: 786-805.

Kyriacou CP, Hall JC (1986). Interspecific genetic control of courtship song production and reception in Drosophila. Science 232: 494-497.

Lande R (1981). The minimum number of genes contributing to quantitative variation between and within populations. Genetics 99: 541-553.

Lindsley DL, Zimm GG (1992). The Genome of Drosophila melanogaster. Academic Press: London.

Moehring AJ, Mackay TF (2004). The quantitative genetic basis of male mating behavior in Drosophila melanogaster. Genetics 167: 1249-1263.

Noor MAF (1997). Genetics of sexual Isolation and courtship dysfunction in male hybrids of Drosophila pseudoobscura and Drosophila persimilis. Evolution 51: 809-815.

Orr HA (1998). The population genetics of adaptation: the distribution of factors fixed during adaptive evolution. Evolution 52: 935-949.

Orr HA (2001). The genetics of species differences. Trends Ecol Evol 16: 343-350.

Orr HA (2005). The genetic theory of adaptation: a brief history. Nat Rev Genet 6: 119-127.

Paallysaho S, Aspi J, Liimatainen JO, Hoikkala A (2003). Role of $X$ chromosomal song genes in the evolution of speciesspecific courtship songs in Drosophila virilis group species. Behav Genet 33: 25-32.

Panhuis TM, Butlin R, Zuk M, Tregenza T (2001). Sexual selection and speciation. Trends Ecol Evol 16: 364-371.

Patterson JT, Stone WS (1952). Evolution in the Genus Drosophila. Macmillan: New York.

Prowell DP (1998). Sex linkage and speciation in Lepidoptera. In: Howard DJ, Berlocher SH (eds) Endless Forms: Species and Speciation. Oxford University Press: Oxford, UK. pp 309-319.

Prum RO (1990). Phylogenetic analysis of the evolution of display behavior in the neotropical manakins (Aves: Pipridae). Ethology 84: 202-231.

Pugh AR, Ritchie MG (1996). Polygenic control of a mating signal in Drosophila. Heredity 77: 378-382.

Reeve HK, Pfennig DW (2003). Genetic biases for showy males: are some genetic systems especially conducive to sexual selection? Proc Natl Acad Sci USA 100: 1089-1094.

Reinhold K (1998). Sex linkage among genes controlling sexually selected traits. Behav Ecol Sociobiol 44: 1-7.

Rice WR (1984). Sex chromosomes and the evolution of sexual dimorphism. Evolution 38: 735-742.

Shaw KL (1996). Polygenic inheritance of a behavioral phenotype: interspecific genetics of song in the Hawaiian cricket genus Laupala. Evolution 50: 256-266.

Shaw KL (2000). Interspecific genetics of mate recognition: inheritance of female acoustic preference in Hawaiian crickets. Evolution 54: 1303-1312.

Sokal RR, Rohlf FJ (1995). Biometry: The Principles and Practice of Statistics in Biological Research. WH Freeman: New York.

Stratton GE, Uetz GW (1986). The inheritance of courtship behavior and its role as a reproductive isolating mechanism in two species of Schizocosa wolf spiders (Araneae; Lycosidae). Evolution 40: 129-141.

Sultana F, Kimura MT, Toda MJ (1999). Anthophilic Drosophila of the elegans species subgroup from Indonesia, with description of a new species (Diptera: Drosophilidae). Entomol Sci 2: 121-126.

Suwito A, Ishida TA, Hattori K, Kimura MT (2002). Environmental adaptations of two flower breeding species of Drosophila from Java, Indonesia. Entomol Sci 5: 399-406. 
Tomaru M, Oguma Y (1994). Genetic basis and evolution of species-specific courtship song in the Drosophila auraria complex. Genet Res 63: 11-17.

True JR (2003). Insect melanism: the molecules matter. Trends Ecol Evol 18: 640-647.

True JR, Weir BS, Laurie CC (1996). A genome-wide survey of hybrid incompatibility factors by the introgression of marked segments of Drosophila mauritiana chromosomes into Drosophila simulans. Genetics 142: 819-837.

True JR, Yeh S-D, Hovemann BT, Kemme T, Meinertzhagen IA, Edwards TN et al (2005). Drosophila tan encodes a novel hydrolase required in pigmentation and vision. PLoS Genet 1: 551-562.

Turelli M, Orr HA (2000). Dominance, epistasis and the genetics of postzygotic isolation. Genetics 154: 1663-1679.

Walter MF, Black BC, Afshar G, Kermabon A-Y, Wright TRF, Biessmann H (1991). Temporal and spatial expression of the yellow gene in correlation with cuticle formation and DOPA decarboxylase activity in Drosophila development. Dev Biol 147: 32-45.

West-Eberhard MJ (1983). Sexual selection, social competition, and speciation. Q Rev Biol 58: 155-183.

Whitman DW, Orsak L, Greene E (1988). Spider mimicry in fruit flies, Diptera Tephritidae. Further experiments on the deterrence of jumping spiders Araneae Salticidae by Zonosemata vittigera Coquillett. Ann Entomol Soc Am 81: 532-536.

Wiens JJ (2000). Decoupled evolution of display morphology and display behaviour in phrynosomatid lizards. Biol J Linn Soc 70: 597-612.

Wittkopp PJ, True JR, Carroll SB (2002). Reciprocal functions of the Drosophila Yellow and Ebony proteins in the development and evolution of pigment patterns. Development 129: 1849-1858.

Wright TRF (1987). The genetics of biogenic amine metabolism, sclerotization, and melanization in Drosophila melanogaster. Adv Genet 24: 127-222. 